\title{
Extinction Distances: Partly New Access to an Old Problem
}

\author{
F. Kerber ${ }^{1}$, C. Kienel $^{1}$, R. Weinberger ${ }^{1}$ and R. Danner ${ }^{2}$ \\ ${ }^{1}$ Institut für Astronomie, Universität Innsbruck; ${ }^{2}$ California Institute of Technology
}

The distance determination to planetary nebulae $(\mathrm{PNe})$ remains a serious problem as illustrated during the last IAU symposium in 1991 (Terzian, IAU 155, p.109). Since then the situation has not improved significantly. The number of stars for which reddening and spectral data are available, though, has increased tremendously over the last decade. Using data from the literature we have determined extinction distances for more than $50 \mathrm{PNe}$. The extinction distance method has been pioneered by Lutz (ApJ 181, 135 (1973)) and Acker (A\&AS 33, 367 (1978)). The basic assumption of the extinction method is that a reddening vs distance relation (RDR) exists along the line of sight to a given object. Once this relation and the reddening of the object itself is known, a distance to the object can be derived. Gathier et al. (A\&A 157, 171 (1986)) present a thorough discussion of the advantages and possible problems of the method. The most recent contributions are by Martin (A\&A 281, 526 (1994)) and Saurer (A\&A 297, 261 (1995)).

Data for stars within a radius of $1^{\circ}$ of the PN were obtained from SIMBAD. Next each field was individually checked on the POSS or ESO/SERC survey and a map of the extinction was derived from the stellar density; stars that belong to the same reddening were extracted and a RDR was established. The use of extinction maps proved to be essential in order to reduce scatter in the RDRs and obtain reliable results. Individual extinction distances could be determined for over 50 objects including a significant number of - mostly limit (e.g. $>5 \mathrm{kpc}$ ) - distances to likely bulge PNe. As a preliminary result we can confirm the existence of the galactic bulge $\mathrm{PNe}$ based on individual distances. The generally assumed value of 80 to $90 \%$ probability of bulge membership for objects with $350^{\circ} \leq 1 \leq$ Additionally extinction distances to about 20 non-bulge PNe have been determined. The complete results will be presented elsewhere in the near future. The use of CCD-photometry will make it possible to derive even more reliable distances. A dedicated campaign to this end is in preparation.

\begin{tabular}{c|c|c|c|c|c} 
Name & Designation & $\oslash\left[{ }^{\prime \prime}\right]$ opt/rad & $\mathrm{E}(\mathrm{B}-\mathrm{V})$ & Dist. [kpc] & other Dist. \\
\hline H 1-36 & PN G353.5-04.9 & $* /$ & 0.40 & $7 \pm 1$ & 2.5 \\
B13-10 & PN G000.1-02.3 & $9.0 /$ & 1.0 & $\geq 6$ & no \\
M2-20 & PN G000.4-01.9 & $6.6 / 16.4$ & 1.2 & $\geq 6$ & no \\
M2-21 & PN G000.7-02.7 & $7.2 /$ & 0.7 & $\geq 6$ & no \\
B13-13 & PN G000.9-02.0 & $5.2 /$ & 1.5 & $\geq 6$ & no \\
M 3-20 & PN G002.1-02.2 & $6.6 /$ & 0.95 & $\geq 8$ & $3.7 / 4.7$ \\
H 1-63 & PN G002.2-06.3 & $7.0 /<2.2$ & 0.41 & $\geq 5$ & 5.7 \\
M 2-38 & PN G005.7-05.3 & $9.3 / 7.4$ & 0.55 & $\geq 7$ & $3.7-6.9$ \\
\hline
\end{tabular}

This research was supported by the "Fonds zur Förderung der wissenschaftlichen Forschung", project no. P10279-AST. This research has made extensive use of the SIMBAD database, operated at CDS, Strasbourg, France. 